\title{
COMENTARIO EDITORIAL Un acercamiento a la investigación cualitativa
}

La investigación cualitativa (IC) tiene su origen a principios del siglo XX y es la antropología la disciplina que inicia su utilización, estudio y sistematización. Recién a partir de los años '30 las escuelas norteamericanas de sociología y psicología inician una amplia historia de estudios cualitativos. Pero en la década del '50, el interés por la IC comienza a decaer debido a la supremacía de lo objetivo y el interés por los números; y es así como se impone la metodología cuantitativa en las ciencias sociales y del comportamiento. Con sus altos y bajos, la IC comienza a ser utilizada por las ciencias de la salud recién en los años '80.

En ocasiones la investigación cualitativa es vista únicamente como un vehículo para generar hipótesis o identificar problemas, para aplicar posteriormente una técnica positivista (método o investigación cuantitativa); no obstante las técnicas cualitativas son útiles en sí mismas con el propósito de encontrar o interpretar las respuestas de las personas o comunidades, intentando identificar el significado sociocultural que un evento dado puede tener en una comunidad.

La investigación cualitativa tiene características distintivas y particulares; siendo el objetivo principal entender a la sociedad desde la perspectiva del sujeto-actor, considerando que la realidad es todo aquello percibido y entendido por las personas. Se preocupa por el significado más que por la medida de los mismos, se centra en los individuos, trata de captar las experiencias de las personas en sus propios términos, palabras y visiones, y asume que la realidad tiene múltiples perspectivas y verdades dependiendo de los diferentes puntos de vista (Tabla 1). En otras palabras, los estudios cualitativos permiten que el investigador examine o explique los problemas o eventos desde la perspectiva del actor, utilizando técnicas no directivas (observación, entrevistas, etc.).

Tabla. 1- Diferentes perspectivas de la IC

\begin{tabular}{|c|c|}
\hline 1. Investigación naturalística & $\begin{array}{l}\text { Estudia situaciones del mundo real tal como se producen naturalmente. No manipula, no controla, es abierta a todo lo que } \\
\text { emeria: "ausencia de resultados predeterminados". }\end{array}$ \\
\hline 2. Análisis inductivo & $\begin{array}{l}\text { Profundiza en los detalles de los datos para descubrir categorías, dimensiones e interrelaciones. Se explora } \\
\text { mediante cuestiones abiertas en lugar de probar teorías derivadas de hipótesis (deductivo). }\end{array}$ \\
\hline 3. Perspectiva holística & $\begin{array}{l}\text { La globalidad del fenómeno en estudio es entendida como un sistema complejo, que es más que la suma de sus partes. } \\
\text { Trata de descubrir interdependencias. No interesa reducir el fenómeno a unas pocas variables o a una relación causa-efecto. }\end{array}$ \\
\hline 4. Datos cualitativos. & $\begin{array}{l}\text { Detallada y profunda descripción (investigación en profundidad). Capta las expectativas y experiencias personales en las } \\
\text { expresiones y palabras de los participantes. }\end{array}$ \\
\hline 5. Contacto personal & $\begin{array}{l}\text { El investigador tiene un contacto directo y cercano con las personas, situaciones o fenómenos objeto de estudio. } \\
\text { Las experiencias personales del investigador son una parte importante en la investigación y en la comprensión del fenómeno. }\end{array}$ \\
\hline 6. Sistemas dinámicos & Se centra en los procesos y asume que los cambios son constantes ya que el foco son los individuos o las culturas. \\
\hline 7. Caso único & $\begin{array}{l}\text { Asume que cada caso es especial y único. El primer nivel de la exploración es ser riguroso, respetando y captando los } \\
\text { detalles de los casos individuales. El análisis final procede y depende de la calidad de los casos individuales estudiados. }\end{array}$ \\
\hline 8. Sensibilidad hacia contexto & $\begin{array}{l}\text { Sitúa los hallazgos en un contexto social, histórico y temporal. Duda de la posibilidad y significado de la generalización } \\
\text { descontextualizada. }\end{array}$ \\
\hline 9. Enfatiza la neutralidad & $\begin{array}{l}\text { La objetividad es imposible. La subjetividad pura altera la credibilidad. El interés del investigador es entender el mundo en toda } \\
\text { su complejidad, no probando nada, sin defender ni avanzar con ideas personales. El investigador adopta una posición } \\
\text { neutral y no enjuiciadora hacia cualquier aspecto que emerja. }\end{array}$ \\
\hline 10. Flexibilidad de diseño & $\begin{array}{l}\text { Abierto, adapta la investigación a cambios de situación. Evita cerrarse en diseños rígidos que eliminan la sensibilidad. } \\
\text { Persigue nuevos caminos cuando emergen. }\end{array}$ \\
\hline
\end{tabular}

Tomada de Patton MQ.

Existen diferencias de raíz entre la investigación cualitativa y cuantitativa. Estas dos metodologías parten de diferentes conceptos y estudian fenómenos distintos, o bien tratan de dar respuestas diferentes de un mismo suceso (ver Tabla 2). Por ejemplo ante un evento particular las preguntas que se plantean son diferentes. Es por esta razón, que en ocasiones será más apropiado aplicar una metodología que la otra y otras veces será de utilidad la combinación de ambas, entendiendo que la mayoría de las veces trata de técnicas complementarias y no excluyentes entre sí.

Tabla. 2- Diferencias entre la investigación cualitativa y cuantitativa.

\begin{tabular}{|c|c|c|}
\hline & Cualitativa & Cuantitativa \\
\hline Problema en estudio & $\begin{array}{l}\text { El problema está poco estudiado y es necesario desarrollar teorías. } \\
\text { El fenómeno no se adecua a medidas cuantitativas (mundo subjetivo) } \\
\text { Estudia el "illness" o respuesta subjetiva de la persona al } \\
\text { hecho de encontrarse mal. }\end{array}$ & $\begin{array}{l}\text { Hay hipótesis que se quieren probar y generalizar. } \\
\text { El fenómeno se adecua a medidas cuantitativas (física, } \\
\text { biología, etc.). } \\
\text { Estudia la "disease" o visión biomédica de la enfermedad. } \\
\text { Estudia relaciones de causalidaud. }\end{array}$ \\
\hline Tipo de estudio & $\begin{array}{l}\text { Inductivo, pretende entender. Contextualiza el fenómeno en } \\
\text { el espacio y tiempo. }\end{array}$ & $\begin{array}{l}\text { Deductivo, pretende probar. Estudia el fenómeno descon- } \\
\text { textualizado, universalinzándolo. }\end{array}$ \\
\hline Objetivo & Interpretación y búsqueda de significado y sentido. & Generalización y búsqueda de leyes universales. \\
\hline Investigador & $\begin{array}{l}\text { Está abierto a todo. Las categorías emergen de los } \\
\text { participantes. }\end{array}$ & Decide las variables a estudiar. \\
\hline Datos & Profundos detallados. Instrumentos flexibles. & Numéricos, objetivos. Instrumentos estandarizados. \\
\hline
\end{tabular}

En cuanto al diseño de un estudio de investigación cualitativa se debe cumplir con ciertos escalones del proceso, y a la hora de plantear un estudio los pasos a seguir son similares a la investigación cuantitativa. Las preguntas que nos debemos responder son: ¿cuál es el objetivo del estudio?; ¿qué información se necesita?; ¿qué tipo de estudio interesa?; ¿cuál será la unidad de análisis?; ¿cómo será la muestra?; ¿qué tipos de datos se recogerán?; ¿ cuál será el método?; ¿cómo se asegurará el rigor?; ¿cuál será la estrategia?.
En este punto particular, la principal diferencia en cuanto a el diseño de un estudio cualitativo en relación a uno cuantitativo, se define bien entendiendo que en este caso el diseño es de carácter único pero flexible, dado que evoluciona constantemente a través del proceso de la investigación; y el sentido de esta flexibilidad se basa en tratar de obtener los datos con la mayor riqueza y detalle posibles. La naturaleza del proceso cualitativo es iterativo, donde el investigador va y vuelve permanentemente del campo. Este proceso es muy intenso, 
requiere mucho tiempo por parte del investigador, y difiere fundamentalmente del concepto cuantitativo ya que los datos obtenidos serán palabras, conductas o acciones. La unidad de análisis son los individuos, grupos, etnias, comunidades, clases en un centro escolar, etc. En la IC el poder no depende del tamaño de la muestra o de la "significancia estadística", sino en qué medida refleja y maximiza la diversidad del fenómeno estudiado. El tamaño de la muestra debe cumplir con el "criterio de saturación"; y para ello se deben responder las siguientes preguntas: ¿los datos obtenidos son suficientes?; ¿han quedado aspectos poco explorados? y ¿los resultados son completos?. Es decir que, la riqueza de la información no es "medible". El investigador cualitativista debe tener siempre presente que no pueden quedar aspectos sin recabar; y que si los datos no aportan más información que la obtenida, se acepta que el tamaño de la muestra es la adecuada y que por lo tanto, cumplió con el criterio de saturación. Para ta recolección de datos y transformación de la información existen diferentes instrumentos. Las técnicas más conocidas para recabar los datos son la entrevista en profundidad (conversación informal, semiestructurada y estructurada), los grupos focales o grupos de discusión, los métodos observacionales y el análisis de documentos escritos. Cada una de las diferentes técnicas mencionadas de recolección de datos tiene una metodología particular a seguir. Éstas se pueden combinar entre sí (por ejemplo realizar grupos focales y posteriormente entrevistas semiestructuradas), pero todas requieren un entrenamiento y una intensa labor por parte del investigador, por lo cual difícilmente un investigador pueda llevar a cabo más de un estudio cualitativo de manera simultánea. El camino que sigue la investigación cualitativa es el siguiente:

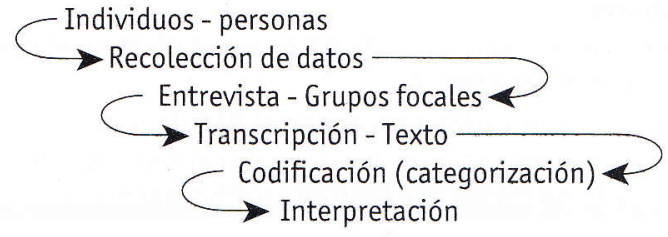

El análisis de los datos no es lineal sino iterativo y generalmente se va procesando a medida que es recolectado. Es fundamental la transcripción en detalle de las notas y los textos. Afortunadamente en la actualidad existen programas informáticos (NUDIST, THE ETHNOGRAPH, LIPSQUAL, etc.) que tienen la finalidad de almacenar, separar, codificar y recuperar los datos cualitativos (amanuenses mecánicos). Es decir que permiten el análisis de las llamadas fuentes no estructuradas, haciendo muçho más amenos y fácil el manejo de los datos para los investigadores.

En la investigación cualitativa existen criterios de rigor a seguir. Quizás

la mayor debilidad que los cuantitivistas le adjudican a la IC es la falta de rigor, si bien que para evaluarla utilizaron sus propios criterios de validación como objetividad, validez y fiabilidad. Por ello, Krefting y col. en 1991 proponen una adaptación de estos criterios a la IC, como se muestra en la Tabla.3, analizando las dos metodologías de investigación.

Tabla. 3- Validez y fiabilidad

\begin{tabular}{c|c}
\hline I. Cualitativa & I. Cuantitativa \\
\hline Credibilidad & Validez interna \\
\hline Aplicabilidad-transferibilidad & Validez externa \\
\hline Consistencia-auditabilidad & Fiabilidad \\
\hline Neutralidad-imparcialidad & Objetividad \\
\hline
\end{tabular}

Krefting L. Rigor in qualitative research: the assessment of trustworthiness. Am J Occup Ther 1991; 45: 214-221.

En IC se dice que un estudio es creíble cuando presenta una descripción detallada de los hechos tal como las personas lo sienten o lo viven; que es aplicable cuando los investigadores maximizan la diversidad en la obtención de la muestra y realizan una descripción detallada del contexto y de los participantes; y consistente cuando otro investigador puede realizar una réplica del mismo, obteniendo resultados similares y nunca contradictorios.

Las principales causas de falta de rigor se deben a la presencia de sesgos. Estos pueden ser sesgos en el muestreo: a) muestra no representativa y b) muestra que no alcanza la saturación (comprometen la transferibilidad y la consistencia). 0 bien, por sesgos en la recolección de datos y en el análisis: a) atribuibles al investigador e b) introducidos por los participantes (comprometen la credibilidad, la consistencia y neutralidad de los hallazgos).

Para controlar los sesgos existen diferentes técnicas. Los métodos utilizados en IC para minimizar los errores son múltiples, sólo mencionaremos aquellos de uso más común: a) verificación por los participantes; b) buscar evidencia de disconformidad con los resultados; c) triangulación y d) descripción detallada.

Las principales aplicaciones de la IC en las ciencias de la salud son: el estudio de hechos, de conceptos y temas poco conocidos que precisan una mayor aclaración (identificar nuevas variables, abrir nuevas hipótesis y generar nuevas ideas). Por otro lado se encuentra la comprensión de aspectos que están en el mundo subjetivo de los individuos, explorando creencias, expectativas y sentimientos, y explicando el porqué de los comportamientos y actitudes.

La fortaleza de la investigación cualítativa está en conocer y entender el mundo subjetivo de las personas, sus familias y comunidades. Hoy en día no es posible concebir un profesional de la salud, y en particular de atención primaria, que no esté preocupado por el sentir-sufrir; por las creencias, actitudes y comportamientos de la población que atiende.

Dras. Vilda Discacciati y Nanci Giraudo

Bibliografia Sugerida

Unidad de Medicina Familiar y Preventiva. Hospital Italiano de Buenos Aires.

1. Britten N. Fisher B. Qualitative research an general practice. British Jorurnal of General Practice 1993; 43: 270-271.

2. Patton MQ. 1990. "Designing Qualitative Studies", Purposeful Sampling. Cap. 5, pp. 169-186, en Qualitative Evaluation and Research Methods.

3. Patton MQ. 1990. "Analysis, Interpretation and Reporting". Cap. 3, pp. 369-506, en Qualitative Evaluation and Research Methods.

4. Taylor SJ. Y Bogdañ R. 1996. "Introducción. Ir hácia la gente'". Cap. 1 en Introd. a los métodos cualitativos de investigación. Paidós, Barcelona, pp. 15-27.

5. Krefting L. Rigor in qualitative research: the assessment of trustworthiness. Am J Occup Ther 1991; 45: 214-221.

6. Crabtree B.F., Miller W.L.1992. Research Methods for Primary Care. Vol 3. "Doing Qualitative Research".

\section{¿Cómo hacemos EVIDENCIA?}

Para informarse de la metodología de realización de la revista Evidencia y la forma en la que debe ser citada, consulte el ejemplar Año 2000 Vol. 3 No 2 Marzo/Abril páginas 36-37.

\section{Fe de eratas, Volumen 3 Numero 4}

1. Página 103. Donde dice (segundo párrafo de la segunda columna del comentario).

"En mayores de dos años con alta probabilidad se recomienda iniciar tratamiento de inmediato sin esperar el cultivo. En pacientes con probabilidad intermedia hacer test rápido y si esto no es posible o difícil el seguimiento, iniciar tratamiento". Debe decir "Teniendo en cuenta el aceptable valor predictivo positivo mencionado previamente, que en Argentina los tests rápidos no siempre están disponibles y que la accesibilidad al sistema de salud dista de ser la ideal (lo que hace prever dificultades en el seguimiento y la reevaluación del paciente), como excepción a la regla podría considerarse la indicación de tratamiento antibiótico sin esperar la documentación de la infección en algunas poblaciones especiales: los pacientes mayores de dos años con alta (o eventualmente probabilidad intermedia) probabilidad clínica de faringitis estreptocócica (más de tres criterios en el primer caso y de dos en el segundo); los que tienen antecedente fiebre reumática y los convivientes de un caso con infección documentada".

2. Se incluyen en el glosario alqunos términos no incluídos en el número anterior.

3. Página 111. Donde dice "Se excluyeron los pacientes 50 años" debe decir "Se excluyeron los pacientes <50 años". 